\title{
Doença arterial periférica em doentes com diabetes nos cuidados de saúde primários: estudo observacional
}

David Brito, ${ }^{1,4}$ Henrique Correia, ${ }^{1,5}$ Ana Vaz Ferreira, ${ }^{1,6}$ Susana Jorge, ${ }^{2,7}$ Hernâni Caniço ${ }^{1,3,6}$

\section{RESUMO}

A doença arterial periférica (DAP) associa-se a risco de ulceração e amputação. A determinação do índice de pressão tornozelo-braço (IPTB) é recomendada para a sua deteção em doentes com diabetes selecionados, associada à avaliação clínica.

Objetivos: Determinar a prevalência das manifestações de DAP em doentes com diabetes nos cuidados de saúde primários (CSP). Local: Centro de Saúde de São Martinho do Bispo.

População: Doentes com diabetes em cuidados de saúde primários.

Tipo de estudo: Estudo observacional, analítico e transversal, que resulta do projeto de intervenção Venha Pelo Seu Pé.

Métodos: Amostra de conveniência de 418 doentes com diabetes ( $48,4 \%$ da população), idade $\geq 50$ anos, observados em consulta de pé diabético. Metodologia e colheita de dados realizadas por profissionais de saúde com experiência. Variáveis: idade, sexo, IMC, perímetro abdominal, pressão arterial, antecedentes tabágicos, dislipidemia, hipertensão, lesões macrovasculares, local de acompanhamento, duração da diabetes, HbA1c registada, terapêutica da diabetes, claudicação arterial e dor em repouso, palpação de pulsos tibiais/pediosos e valores dos IPTB. Usaram-se os testes qui-quadrado ou de Fisher na análise de associação de variáveis.

Resultados: Quatrocentos e dezoito doentes com idade média de $68,85 \pm 8,64$ anos, $52,9 \%$ do sexo feminino. Com $2,9 \%$ de fumadores, $84,0 \%$ com dislipidemia e $87,1 \%$ hipertensos (ou terapêutica para esses problemas) e $19,1 \%$ com lesões macrovasculares. Duração média da diabetes de $10,72 \pm 8,48$ anos e média de HbA1c de $6,86 \pm 1,16 \%$. Proporção de doentes com úlcera, e amputados, de 0,5\%. A prevalência de claudicação arterial foi de 9,8\% (IC95\%: 7,1-13,1), de pulso não palpável de 14,6\% (IC95\%: 11,3-18,3) e IPTB $\leq 0,9$ de 11,5\% (IC95\%: 6,6-14,9), com prevalência global de alterações sugestivas de DAP de 28,5\% (IC95\%: 24,2-33,1). Foi identificada associação significativa entre IPTB $\leq 0,9$ e pulso não palpável $(p=0,009)$ ou claudicação arterial $(p<0,001)$ na análise por doente. Conclusões: Não é possível concluir comparativamente com outros estudos quanto à prevalência de DAP, dada a escassez da determinação de IPTB em estudos de doentes com diabetes nos CSP portugueses. Outros estudos parecem sugerir tratar-se de uma prevalência inferior ou subdiagnóstico. A complementaridade de métodos poderá contribuir para maior deteção de casos na prática clínica.

Palavras-chave: Pé diabético; Doença arterial periférica; Índice tornozelo-braço; Claudicação intermitente; Cuidados de saúde primários

\section{INTRODUÇÃO}

$\mathrm{A}$ doença arterial periférica (DAP) é uma patologia identificada no pé diabético e é fator de risco para ulceração e amputação. A sua prevalência em doentes com diabetes mellitus tipo 2 é frequentemente subestimada, dada a sua ocorrência em doentes assintomáticos ou com sintomas atípicos e a variedade de critérios e metodologia diagnóstica. A prevalência nessa população é superior à da população em geral e tende a aumentar com a idade e com fatores de risco adicionais. ${ }^{1}$ Estima-se que seja superior a $20 \%$ em doentes com diabetes ${ }^{1}$ e cerca de $50 \%$ destes com úlcera dos membros inferiores de novo, ${ }^{2} \mathrm{es}-$ tando associada a morbimortalidade cardiovascular. ${ }^{1} \mathrm{O}$ reconhecimento da DAP em cuidados de saúde primários (CSP) é reduzido, ${ }^{3}$ tornando-se especialmente importante nos doentes com diabetes, dado que se inicia precocemente, progride rapidamente, é grave e extensa e apenas sintomática em fases mais avançadas. ${ }^{1}$ Além disso, a DAP nesses doentes não

1 Médico Especialista em Medicina Geral e Familiar

2 Enfermeira em Cuidados de Saúde Primários | Pós-graduada em Diabetes

3 Doutorado em Ciências da Saúde | Medicina Familiar e Comunitária

4 USF VitaSaurium | Soure

5 USF Progresso e Saúde | Tocha | Cantanhede

6 UCSP Dr. Manuel Cunha | São Martinho do Bispo | Coimbra

7 USF Mondego | São Martinho do Bispo | Coimbra 
é muitas vezes revascularizável, fruto da sua distribuição multisegmentar e distal, ${ }^{1}$ exigindo uma ação preventiva precoce.

Vários métodos têm sido estudados e utilizados no rastreio e diagnóstico da patologia arterial dos membros inferiores. A história clínica e o exame físico podem sugerir o diagnóstico de DAP e a sua avaliação de rotina no doente com diabetes é recomendada em várias orientações, ${ }^{4-5}$ incluindo aquelas emitidas pela Direção-Geral da Saúde (DGS) $\cdot^{6-7}$ No entanto, a acuidade diagnóstica para DAP através de sintomas, sinais ou pela palpação de pulsos periféricos é baixa, o que resulta também das baixas sensibilidades desses métodos. ${ }^{8}$

Alguns métodos para deteção de DAP com recurso a instrumentos de utilização na prática clínica, como a determinação do índice de pressão tornozelo-braço (IPTB), têm sido recomendados ${ }^{4-5}$ ainda que a evidência da utilidade da sua utilização sistemática seja parca. Outros métodos, como a determinação da pressão sistólica no hálux ou o uso qualitativo de doppler de onda contínua, têm também sido estudados para esse fim. ${ }^{1}$ Várias questões têm sido levantadas relativamente à aplicabilidade do IPTB no diagnóstico de DAP em doentes com diabetes, devido à sua variação em doentes hipo/hipertensos e à presença de calcificação arterial (arterioesclerose de Mönckeberg). O fenómeno de arteriosclerose em doentes com diabetes coloca em causa a utilidade do limiar diagnóstico de DAP mais comumente utilizado por esse método (IPTB $\leq 0,9$ ), especialmente aqueles com lesões no pé, neuropatia, nefropatia e elevado risco cardiovascular. Existe também uma franja de doentes com diabetes com valor de IPTB elevado $(>1,3-1,4)$ a que corresponde o mesmo fenómeno de calcificação e que, ainda assim, apresentam algum grau de compromisso arterial. Apesar dessas limitações, um valor de IPTB $\leq 0,9(e>1,4)$ está associado a pior prognóstico em termos de morbimortalidade cardiovascular e amputação. ${ }^{9}$ A determinação do IPTB na prática clínica dos CSP levanta ainda questões relacionadas com a viabilidade e adequação da sua utilização, a correção metodológica e a necessidade de treino. ${ }^{8,10}$

Numa população com diabetes, em contexto de CSP, definiu-se como objetivo determinar a prevalência das manifestações de DAP utilizando critérios clínicos e resultados da avaliação do IPTB.

\section{MÉTODOS}

Este estudo deriva de dados obtidos no projeto de intervenção em saúde Venha Pelo Seu Pé, desenvolvido pela ONGD Saúde em Português e apoiado pela DGS durante o ano de 2012, em que foi desenvolvido e aplicado um modelo de cuidados multidisciplinares dedicado ao pé diabético em CSP.
Trata-se de um estudo observacional, analítico e transversal. A amostra de conveniência é constituída por 418 doentes com diabetes com idade igual ou superior a 50 anos, de uma população total de 864 com diabetes do Centro de Saúde de São Martinho do Bispo elegíveis para integrar o projeto. Os doentes acederam ao convite informal dos médicos e enfermeiros de família dessa unidade de saúde para participar numa consulta de pé diabético no âmbito do projeto Venha Pelo Seu Pé, entre março e dezembro de 2012. Foram excluídos doentes com registo de dados incompleto, dados desconhecidos ou que responderam inconclusivamente à entrevista clínica.

A metodologia de recolha de dados e de avaliação de variáveis foi preparada. Foi realizada uma reunião inicial de uniformização de procedimentos e metodologias, incluindo a apresentação e discussão da metodologia de obtenção do IPTB, dirigida a todos os médicos e enfermeiros diretamente envolvidos, partindo da discussão de resultados de um inquérito interno e respetiva correção. Foram recolhidas as variáveis idade (anos), sexo, índice de massa corporal (IMC) $\left(\mathrm{kg} / \mathrm{m}^{2}\right)$, perímetro abdominal (cm), pressão arterial ( $\mathrm{mmHg}$ ), antecedentes tabágicos, dislipidemia (diagnóstico prévio ou terapêutica atual com estatina), hipertensão (diagnóstico prévio ou terapêutica atual com anti-hipertensor de qualquer classe), lesão macrovascular (i.e., acidente vascular cerebral ou isquémico transitório, angina ou enfarte do miocárdio, DAP dos membros inferiores ou carotídea, ou placas de ateroma, conhecida ou registada), local de acompanhamento clínico da diabetes, duração da diabetes (anos) desde o diagnóstico, valor de HbAlc (\%) registado nos últimos seis meses e terapêutica da diabetes (antidiabéticos orais [ADO] ou insulina). Foram ainda registadas as variáveis: sintomas de doença arterial dos membros inferiores (claudicação arterial ou dor gemelar em repouso), objetivação da palpação de pulsos pedioso dorsal e tibial posterior e índices de pressão tornozelo-braço (relação entre pressão sistólica com doppler do tornozelo/pé e pressão sistólica braquial com doppler).

Os dados foram obtidos através da consulta do registo clínico, colheita de história clínica (antecedentes pessoais e duração da diabetes) e medição em consulta (peso em balança analógica e altura com estadiómetro fixo, com doente descalço e vestido, perímetro abdominal na linha horizontal intermédia à altura crista ilíaca - grelha costal, pressão arterial com dispositivo de medição oscilométrica automático, exame físico e IPTB). O IPTB foi determinado com doente em posição supina ou semissentado após alguns minutos, utilizando dopplervascular portátil de onda contínua (artérias pediosa dorsal, tibial posterior e radial), esfigmomanómetro manual e seleção das pressões obtidas mais ele- 
vadas, como descrito noutro artigo. ${ }^{9}$ As avaliações foram efetuadas pelos quatro médicos e/ou quatro enfermeiras do projeto, com experiência na avaliação de doentes com diabetes, incluindo parâmetros objetivos do pé, sem prática de rotina ou treino específico na obtenção do IPTB.

A estatística foi realizada através do software IBM SPSS, v. 20.0, utilizando intervalos de confiança a 95\% calculados para proporções e os teste do qui-quadrado e exato de Fisher para testar associação de variáveis com nível de significância de 0,05 . Os diagramas de Venn proporcionais foram criados com ferramenta euler $A P E$, v. 3.

\section{RESULTADOS}

Foram incluídos 418 doentes com diabetes $(48,4 \%$ da população) que apresentavam uma média de idades de $68,85 \pm 8,64$ anos. A maioria $(52,9 \%)$ era do sexo feminino (Quadro I).

No que se refere aos antecedentes clínicos dos doentes da amostra destacou-se uma proporção de $29,4 \%$ com antecedentes tabágicos. No total de doentes, $87,1 \%$ apresentavam diagnóstico prévio de hipertensão arterial ou encontravam-se medicados com algum tipo de anti-hipertensor e $84,0 \%$ tinham diagnóstico prévio de dislipidemia ou encontravam-se sob terapêutica com estatina. Apenas 19,1\% referiram ou apresentaram registo clínico compatível com lesão macrovascular diagnosticada (Quadro I).

A duração estimada do diagnóstico de diabetes, de acordo com informação reportada, foi em média de 10,72 $\pm 8,48$ anos. A quase totalidade $(96,7 \%)$ dos doentes era acompanhada por motivo de controlo metabólico da diabetes exclusivamente ao nível dos CSP. Observando a terapêutica dirigida à diabetes verificou-se que 94,0\% encontravam-se medicados com antidiabéticos orais e 15,6\% com algum tipo de insulina. No total de doentes, 1,9\% não realizavam qualquer terapêutica farmacológica antidiabética. Relativamente ao controlo metabólico (do qual existia pelo menos um registo nos últimos seis meses) verificou-se um nível médio de HbAlc de 6,86₫1,16\% (Quadro I).

Verificou-se que apenas $0,5 \%$ dos doentes apresentavam úlcera do membro inferior no momento do contacto e que a mesma proporção de doentes tinha sido submetido a algum tipo de amputação a esse nível.

Quanto às características clínicas e de exame objetivo dos doentes verificou-se que a amostra apresentava uma média de valores de $30,51 \pm 4,79 \mathrm{~kg} / \mathrm{m}^{2}$ de IMC, $103,96 \pm 12,23 \mathrm{~cm}$ de perímetro abdominal e 142,80 $\pm 19,52 \mathrm{mmHg}$ de pressão arterial sistólica (Quadro II).

Evidencia-se também que apenas $11,2 \%$ dos doentes referiram sintomatologia compatível com DAP, nomeadamen-
QUADRO I. Características demográficas e antecedentes clínicos

\begin{tabular}{|c|c|}
\hline Amostra ( $n[\%])$ & $418(100)$ \\
\hline \multicolumn{2}{|l|}{ Idade (anos) } \\
\hline 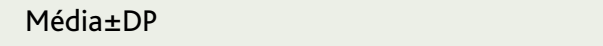 & $68,85 \pm 8,64$ \\
\hline Mínima - Máxima & $50-91$ \\
\hline \multicolumn{2}{|l|}{ Sexo (n [\%]) } \\
\hline Masculino & $197(47,1)$ \\
\hline Feminino & $221(52,9)$ \\
\hline \multicolumn{2}{|l|}{ Antecedentes tabágicos ( $n[\%])$} \\
\hline Fumador & $12(2,9)$ \\
\hline Ex-fumador & $111(26,6)$ \\
\hline Não & $295(70,6)$ \\
\hline \multicolumn{2}{|l|}{ Antecedentes cardiovasculares ( $n$ [\%]) } \\
\hline Hipertensão arterial (ou anti-hipertensor) & $364(87,1)$ \\
\hline Dislipidemia (ou estatina) & $351(84,0)$ \\
\hline Lesão macrovascular & $80(19,1)$ \\
\hline \multicolumn{2}{|l|}{ Diabetes } \\
\hline \multicolumn{2}{|l|}{ Duração da diabetes (anos) } \\
\hline Média $\pm D P$ & $10,72 \pm 8,48$ \\
\hline Mínima - Máxima & $1-44$ \\
\hline \multicolumn{2}{|l|}{ Local de acompanhamento ( $n[\%])$} \\
\hline Cuidados de Saúde Primários & $404(96,7)$ \\
\hline Cuidados de Saúde Primários e Secundários & $14(3,3)$ \\
\hline \multicolumn{2}{|l|}{ Terapêutica antidiabética ( $\boldsymbol{n}[\%]$ ) } \\
\hline ADO & $345(82,5)$ \\
\hline ADO e insulina & $48(11,5)$ \\
\hline Insulina & $17(4,1)$ \\
\hline Não & $8(1,9)$ \\
\hline \multicolumn{2}{|l|}{ Controlo metabólico (HbA1c [\%]) } \\
\hline Média $\pm D P$ & $6,86 \pm 1,16$ \\
\hline \multicolumn{2}{|l|}{ Antecedentes patológicos (outros) ( $n$ [\%]) } \\
\hline \multicolumn{2}{|l|}{ Úlcera do MI } \\
\hline Atual & $2(0,5)$ \\
\hline Prévia & $9(2,2)$ \\
\hline Não & $407(97,4)$ \\
\hline \multicolumn{2}{|l|}{ Amputação dos MI (qualquer) } \\
\hline Bilateral & $1(0,2)$ \\
\hline Unilateral & $1(0,2)$ \\
\hline Não & $416(99,5)$ \\
\hline
\end{tabular}

Abreviaturas: DP = Desvio-Padrão; $\mathrm{MI}=$ Membro(s) inferior(es); $\mathrm{ADO}=$ Antidiabéticos orais.

te claudicação arterial $(9,8 \%)$ e dor gemelar em repouso $(1,4 \%)$, sendo a primeira manifestamente predominante. No que diz respeito à palpação dos pulsos pediosos e tibiais pos- 
teriores verificou-se que em $14,6 \%$ dos doentes não foi detetado pelo menos um pulso num ou em ambos os membros. Da determinação do IPTB resultou um índice $\leq 0,9$ em pelo menos um membro em $11,5 \%$ dos doentes e um índice $>1,4$ em $10 \%$ dos doentes (Quadro II). Relativamente a doentes com índices $\leq 0,97,9 \%$ apresentavam oclusão ligeira (índice $0,7-0,9$ ), $3,6 \%$ oclusão moderada (índice $0,4-0,69$ ), não existindo na amostra doentes com oclusão grave (índice $<0,4$ ).

Comparando dados referentes a aspetos clínicos e objetivos da avaliação vascular realizada e sua interseção por doente observado verifica-se que em mais de metade dos doentes com IPTB reduzido, em pelo menos um membro, foram detetados exclusivamente por esse meio (6,5\% face a $11,5 \%$ com alterações) (Quadro III). O mesmo se observa relativamente à ocorrência de queixas de claudicação arterial ( $5,5 \%$ face a 9,8\% com claudicação arterial) e à objetivação de pelo menos um pulso não palpável (10,5\% face a $14,6 \%$ com pulsos não palpáveis), que apresentavam exclusivamente essas manifestações de DAP. A avaliação de pulsos e de claudicação arterial permitiu detetar 92 (22,0\%) doentes com alterações. Considerando ainda 27 (6,5\%) doentes com alterações exclusivamente no IPTB, obtém-se uma prevalência de alterações sugestivas de DAP de $28,5 \%$.

O diagrama representado na Figura 1 permite observar de forma mais detalhada e compreensível as proporções relativas dos diferentes achados relativos a DAP nos $119(28,5 \%)$ doentes que apresentaram resultados anormais e correspondente sobreposição da sintomatologia com os achados objetivos e complementares. Apenas seis doentes apresentaram parâmetros de doença arterial concordantes nos três aspetos considerados, representando apenas $5,0 \%$ dos doentes com achados alterados.

Verificou-se existir associação entre IPTB $\leq 0,9$ e as variáveis pulso não palpável $(p=0,009)$ e claudicação arterial $(p<0,001)$ quando analisadas por doente, independentemente do membro avaliado. No entanto, na análise por membro afetado, essa associação apenas se verificou entre IPTB $\leq 0,9$ e claudicação arterial (esquerda $p=0,001$ e direita $p<0,001)$. Algumas associações de variáveis apresentaram significado estatístico de forma assimétrica entre membros (apenas à direita, pulso não palpável e IPTB $\leq 0,9$ [ $p=0,043]$ ou claudicação arterial $[p=0,043])$.

\section{DISCUSSÃO E CONCLUSÕES}

Este estudo identificou uma prevalência de doentes com diabetes com critérios sugestivos de DAP (clínica, exame objetivo ou IPTB) de 28,5\% (excluindo doentes com IPTB $>1,4$, considerados nalguns estudos como equivalentes). A comparação com outros estudos não pode ser realizada de forma direta, dadas as diferentes constituições amostrais e os diferentes critérios e meios utilizados no diagnóstico.

A amostra participante deste estudo difere na sua composição de um estudo de prevalência de complicações da diabetes com 4.583 doentes ao nível dos CSP portugueses e tendo por base a Rede Médicos-Sentinela, que apenas reportou antecedentes de bypass arterial periférico presentes em $0,5 \% .{ }^{11}$

Relativamente à prevalência de achados compatíveis com DAP em doentes com diabetes em CSP, outro estudo português realizado nesse nível de cuidados, com vista à caracterização da neuropatia, identificou em 93 doentes com diabetes uma prevalência de $18 \%$ de claudicação intermitente 


\begin{tabular}{|c|c|c|}
\hline $\begin{array}{l}\text { QUADRO III. Distribuição de características clínicas relativa } \\
\text { inferiores }\end{array}$ & arterial dc & nembros \\
\hline Amostra ( $n[\%])$ & $418(100)$ & IC $95 \%(\%)$ \\
\hline Doentes com alterações: & & \\
\hline IPTB $\leq 0,9$ & $48(11,5)$ & $8,6-14,9$ \\
\hline Claudicação arterial & $41(9,8)$ & $7,1-13,1$ \\
\hline Pulso(s) tibial e/ou pedioso não palpável(eis) & $61(14,6)$ & $11,3-18,3$ \\
\hline Global & $119(28,5)$ & $24,2-33,1$ \\
\hline Doentes com alterações exclusivas (apenas um parâmetro al & & \\
\hline IPTB $\leq 0,9$ & $27(6,5)$ & $4,3-9,3$ \\
\hline Claudicação arterial & $23(5,5)$ & $3,5-8,1$ \\
\hline Pulso(s) tibial e/ou pedioso não palpável(eis) & $44(10,5)$ & $7,7-13,9$ \\
\hline Doentes com alterações simultâneas & & \\
\hline IPTB $\leq 0,9$ e claudicação arterial e pulso(s) não palpável(eis) & $6(1,4)$ & $0,5-3,1$ \\
\hline Doentes sem alterações & & \\
\hline IPTB $\leq 0,9$ ou claudicação arterial ou pulso(s) não palpável(eis) & $299(71,5)$ & $66,9-75,8$ \\
\hline
\end{tabular}

avaliada por questionário, distando dos $9,8 \%$ identificados na nossa amostra. Contudo, apesar de a amostra naquele estudo apresentar idade $(65,4 \pm 10,1)$ e duração da diabetes médias $(10,1 \pm 11,1)$, semelhantes a este estudo $(68,85 \pm 8,64$ e $10,72 \pm$ 8,48), existem várias diferenças. Destacam-se a prevalência de tabagismo $(7,5 \%)$ e média de HbAlc $(8,3 \pm 1,9 \%)$, mais elevadas face à nossa amostra $(2,9 \%$ de

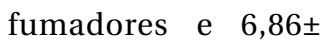
$1,16 \%$ de HbAlc) e também a prevalência de antecedentes de úlcera

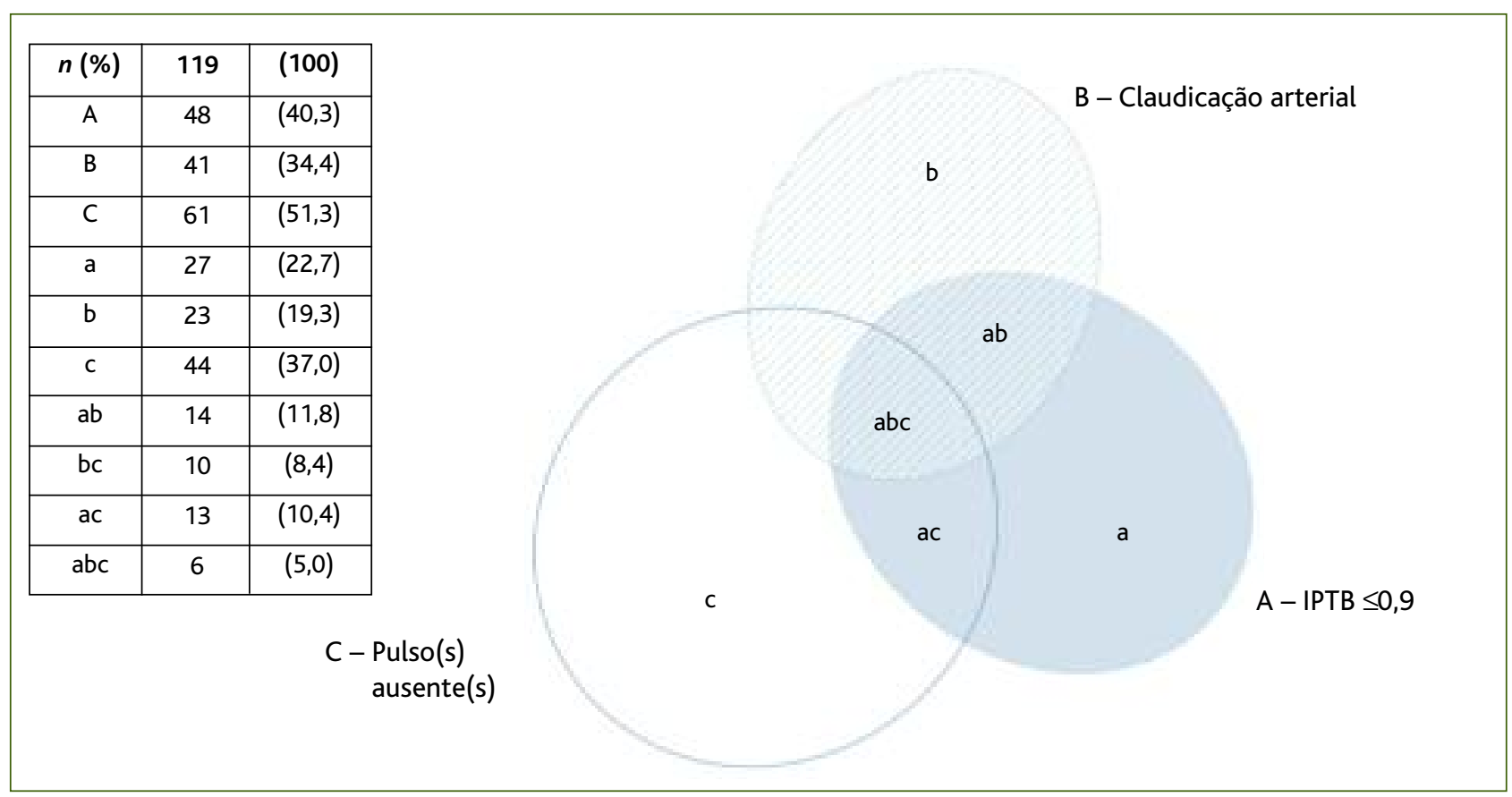

Figura 1. Diagrama de distribuição dos doentes com características clínicas de doença arterial periférica dos MI.

Legenda: $A$ = Doentes com IPTB $\leq 0,9 ; B$ = Doentes com claudicação arterial; $C=$ Doentes com pulso(s) ausente(s). a, b, c = Doentes com alterações exclusivas em um parâmetro dos referidos em $A, B$ e $C$, respetivamente. ab, bc, ac = Doentes com alterações simultâneas em dois dos parâmetros referidos em $A, B$ e $C$. Abc $=$ Doentes com alterações simultâneas nos três parâmetros referidos em $A, B$ e $C$. 
dos membros inferiores $(7,5 \%)$, que poderão estar relacionados com maior prevalência de DAP e que, no presente estudo, foi de $2,6 \%$. No entanto, aquele estudo excluiu doentes sem pulsos palpáveis nos membros inferiores. ${ }^{12}$

Por fim, um estudo português aleatorizado com 237 doentes com diabetes em CSP, em que 97,5\% dos doentes tinham idade superior a 40 anos e proporção equiparável de sexos, observou 3,4\% de doentes com história de amputação ou ulceração e 5,9\% com ambos os pulsos (tibial posterior e pedioso) não palpáveis. Contudo, excluiu doentes com úlcera atual e amputação bilateral dos membros inferiores. Não foram, além disso, referidos alguns fatores de risco (tabagismo e antecedentes de doença cardiovascular) e outros aspetos são de difícil comparação quantitativa. ${ }^{13}$ Esses valores distam dos $2,3 \%$ de doentes com amputação (unilateral) ou úlcera prévia do nosso estudo e sobretudo dos 14,9\% de doentes com pelo menos um pulso não palpável, evidenciando clara diferença de critérios, menos estritos no presente estudo.

Não identificámos outros estudos publicados com amostra de doentes com diabetes ao nível dos CSP, realizados em Portugal, com a utilização da avaliação do IPTB para identificação de DAP.Vários estudos com populações espanholas com diabetes em CSP referem prevalências de IPTB $\leq 0,9$ que variam de 11,3 a 29,3\%, diferindo nas considerações metodológicas e amostras constituídas. ${ }^{14-15}$ Não sendo totalmente equiparáveis à amostra utilizada neste estudo, dados os diferentes valores encontrados para fatores que se sabe influenciarem o seu resultado, como a idade, o tabagismo, o controlo metabólico e a duração da diabetes, entre outros, ${ }^{1}$ poderão sugerir uma menor prevalência nesta população ou algum subdiagnóstico.

A complementaridade de métodos de diagnóstico poderá contribuir para maior deteção de casos de DAP, conforme seria expectável, dadas as limitações na sensibilidade dos métodos utilizados (IPTB, claudicação e pulsos). Neste estudo, nos doentes com alterações sugestivas de DAP, a utilização de um método acrescido à combinação dos outros dois - claudicação arterial, IPTB reduzido e pulsos não palpáveis - permitiu um aumento na deteção de 19,3, 22,7 e 37,0 pontos percentuais, respetivamente. Esses aspetos reforçam a necessidade de estudos dirigidos à avaliação das características e acuidade diagnóstica desses métodos (isolados e em combinação) e também à sua aplicabilidade na prática clínica, não sem antes avaliar a aferição e validade dos instrumentos e métodos que existem nos CSP portugueses em contexto de consulta de pé diabético, por forma a permitir retirar conclusões para o contexto real.

Este estudo apresenta possíveis limitações, de que se destacam o método de amostragem, o critério de inclusão, a va- lidade instrumental (dispositivos utilizados) e metodológica (diagnóstico de claudicação intermitente, posição não supina e tempo de repouso prévio à avaliação do IPTB, ou variabilidade entre avaliadores), a falta de experiência e treino na realização do IPTB, eventualmente a análise de dados e interpretação (variáveis não consideradas e com influência nos resultados, como a neuropatia), entre outras. Esses erros e vieses advêm também da natureza empírica e não experimental do projeto. Por outro lado, tem a mais-valia da dimensão relevante da amostra, constituída por indivíduos selecionados de acordo com recomendações internacionais para a realização de IPTB e da apresentação de resultados da aplicação desse método, pouco reportados na literatura referente aos CSP portugueses. Contribui ainda para alertar para as dificuldades e limitações dos métodos de diagnóstico utilizados e aspetos relevantes relativos à comparabilidade de resultados com outros estudos.

\section{AGRADECIMENTOS}

Os autores agradecem a todos os envolvidos no projeto, sobretudo aos médicos e enfermeiras do Centro de Saúde de São Martinho do Bispo que colaboraram na investigação.

\section{REFERÊNCIAS BIBLIOGRÁFICAS}

1. Jude EB, Eleftheriadou I, Tentolouris N. Peripheral arterial disease in diabetes: a review. Diabet Med. 2010;27(1):4-14.

2. Prompers L, Huijberts $M$, Apelqvist J, Jude E, Piaggesi A, Bakker K, et al. High prevalence of ischaemia, infection and serious comorbidity in patients with diabetic foot disease in Europe: baseline results from the Eurodiale study. Diabetologia. 2007;50(1):18-25.

3. Hirsch AT, Criqui MH, Treat-Jacobson D, Regensteiner JG, Creager MA, Olin $\mathrm{JW}$, et al. Peripheral arterial disease detection, awareness, and treatment in primary care. JAMA. 2001;286(11):1317-24.

4. Hinchliffe RJ, Brownrigg JR, Apelqvist J, Boyko EJ, Fitridge R, Mills JL, et al. IWGDF Guidance on the diagnosis, prognosis and management of peripheral artery disease in patients with foot ulcers in diabetes [Internet]. International Working Group on the Diabetic Foot; 2015 [cited 2015 Dec 1]. Available from: http://www.iwgdf.org/files/2015/website_pad.pdf

5. American Diabetes Association. Standards of medical care in diabetes 2014. Diabetes Care. 2014;37 Suppl 1:S14-80.

6. Direção-Geral da Saúde. Diagnóstico sistemático do pé diabético: norma n 005/2011, de 21/01/2011. Lisboa: DGS; 2011.

7. Direção-Geral da Saúde. Organização de cuidados, prevenção e tratamento do pé diabético: orientação nº 003/2011, de 21/01/2011. Lisboa: DGS; 2011.

8. Nicolaï SP, Kruidenier LM, Rouwet EV, Bartelink ML, Prins MH, Teijink JA. Ankle brachial index measurement in primary care: are we doing it right? Br J Gen Pract. 2009;59(563):422-7.

9. Potier L, Abi Khalil C, Mohammedi K, Roussel R. Use and utility of ankle brachial index in patients with diabetes. Eur J Vasc Endovasc Surg. 2011;41(1):110-6.

10. Davies JH, Kenkre J, Williams EM. Current utility of the ankle-brachial in$\operatorname{dex}(\mathrm{AB})$ in general practice: implications for its use in cardiovascular disease screening. BMC Fam Pract. 2014;15:69. 
11. Falcão IM, Pinto C, Santos J, Fernandes ML, Ramalho L, Paixão E, et al. Estudo da prevalência da diabetes e das suas complicações numa coorte de diabéticos portugueses: um estudo na Rede Médicos-Sentinela [The prevalence of diabetes and diabetic complications in a cohort of portuguese patients: a medical sentinel practice network study]. Rev Port Clin Geral. 2008;24(6):679-92. Portuguese

12. Barbosa AP, Medina JL, Ramos EP, Barros HP. Prevalence and risk factors of clinical diabetic polyneuropathy in a Portuguese primary health care population. Diabetes Metab. 2001;27(4 Pt 1):496-502.

13. Macedo A, Campos C, Correia J, Gomes P. Pé em risco aumentado de ulceração em doentes com diabetes mellitus tipo 2 [Increased risk of foot ulcers in patients with type 2 diabetes mellitus]. Rev Port Clin Geral. 2010;26(2):159-68. Portuguese

14. Mancera-Romero J, Paniagua-Gómez F, Matos-Cerezuela I, Baca-Osorio A, Ruiz-Vera S, González-Santos P, et al. Enfermedad arterial periférica oculta en población diabética seguida en atención primaria [Occult peripheral arterial disease in patients with type 2 diabetes in primary care]. Clin Investig Arterioscler. 2010;22(4):154-61. Spanish

15. Novo-García C, Ciria-Uriel J, Novo-García E, Niño-de-Mateo M. Determinación del índice tobillo-brazo mediante doppler portátil y tensiómetro automático en pacientes diabéticos [Determination of ankle-brachial index using a portable doppler and a blood pressure measuring device in diabetic patients]. Enferm Clin. 2012;22(4):198-204. Spanish

\section{CONFLITOS DE INTERESSE E FINANCIAMENTO}

Este estudo decorre do projeto Venha Pelo Seu Pé, desenvolvido pela ONGD Saúde em Português e apoiado financeiramente pela Direção Geral da Saúde. Todos os autores foram colaboradores ou membros da ONGD Saúde em Português.

\section{COMISSÃO DE ÉTICA}

Estudo realizado com parecer favorável da Comissão de Ética da Unidade de Investigação em Ciências da Saúde: Enfermagem (Escola Superior de Enfermagem de Coimbra).

\author{
ENDEREÇO PARA CORRESPONDÊNCIA \\ David Brito \\ USF VitaSaurium \\ Cruz-Nova \\ 3130-200 Soure \\ Email: davidbrito.mgf@gmail.com
}

Recebido em 25-04-2016

Aceite para publicação em 10-07-2017

\section{ABSTRACT}

\section{PERIPHERAL ARTERIAL DISEASE IN PATIENTS WITH DIABETES IN PRIMARY CARE: AN OBSERVATIONAL STUDY}

Peripheral arterial disease (PAD) puts patients at risk for skin ulceration and limb amputation. Measurement of the ankle-brachial in$\operatorname{dex}(\mathrm{ABI})$ along with clinical evaluation to detect PAD is recommended for selected diabetic patients.

Objectives: To determine the prevalence of PAD signs in diabetic patients managed in primary care.

Setting: Centro de Saúde de São Martinho do Bispo.

Participants: Diabetic patients managed in primary care in this clinic.

Study type: Observational study, using data obtained from the «Venha Pelo Seu Pé project».

Methods: A convenience sample of diabetic patients was selected, aged 50 years of age or over, attending a diabetic foot care consultation. Data were collected by experienced healthcare professionals working in this clinic. Data were obtained in an interview, by physical examination, and from clinic records. These included age, sex, body mass index (BMI), waist circumference, blood pressure, past medical history of tobacco use, dyslipidemia, hypertension, macrovascular disease, duration of diabetes, last noted value of glycosylated haemoglobin ( $\mathrm{HbA} 1 \mathrm{c})$, diabetes medication, arterial claudication and resting pain, palpation of pedal pulses, and ABI values. The chi-square and Fisher tests were used to test associations between variables.

Results: There were 418 patients in the study sample comprising $48.4 \%$ of the diabetic patients in this clinic. The mean age was $68.85 \pm 8.64$ years and $52.9 \%$ were females. In the sample, $2.9 \%$ were smokers, $87.1 \%$ had hypertension and $84.0 \%$ had dyslipidemia (or medication for those problems). In 19.1\% there was known macrovascular disease. Diabetes had been known for a mean duration of $10.72 \pm 8.48$ years and the mean $\mathrm{HbA} 1 \mathrm{c}$ was $6.86 \pm 1.16 \%$ of. The foot ulcer, or amputation, was found in $0.5 \%$. Arterial claudication was present in 9.8\% (Cl95\%: 7.1-13.1) and a non-palpable pulse in 14.6\% (Cl95\%: 11.3-18.3). The ABI was less than or equal to 0.9 in $11.5 \%$ (CI95\%: 6.6-14.9), with a prevalence of clinical findings of PAD of $28.5 \%$ (CI95\%: 24.2-33.1). An association was found between $A B I \leq 0.9$ and a non-palpable pulse $(p=0.009)$ or arterial claudication $(p<0.001)$.

Conclusions: We cannot compare the prevalence of PAD in this study with findings from other Portuguese studies, because $A B I$ was measured in few studies. The findings in other studies suggest that there might be a lower prevalence or under-diagnosis of PAD. A combination of different diagnostic methods may contribute to increase in the detection of PAD in clinical practice.

Keywords: Diabetic foot; Peripheral arterial disease; Ankle brachial index; Intermittent claudication; Primary health care 